\title{
Nanoscale
}

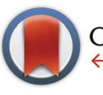

CrossMark \& click for updates

Cite this: Nanoscale, 2016, 8, 14556

\section{Showing particles their place: deterministic colloid immobilization by gold nanomeshes $\uparrow$}

\author{
Christian Stelling, ${ }^{a}$ Andreas Mark, ${ }^{b}$ Georg Papastavrou* ${ }^{\star b}$ and Markus Retsch*a
}

The defined immobilization of colloidal particles on a non-close packed lattice on solid substrates is a challenging task in the field of directed colloidal self-assembly. In this contribution the controlled selfassembly of polystyrene beads into chemically modified nanomeshes with a high particle surface coverage is demonstrated. For this, solely electrostatic interaction forces were exploited by the use of topographically shallow gold nanomeshes. Employing orthogonal functionalization, an electrostatic contrast between the glass surface and the gold nanomesh was introduced on a sub-micron scale. This surface charge contrast promotes a highly site-selective trapping of the negatively charged polystyrene particles from the liquid phase. AFM force spectroscopy with a polystyrene colloidal probe was used to rationalize this electrostatic focusing effect. It provides quantitative access to the occurring interaction forces between the particle and substrate surface and clarifies the role of the $\mathrm{pH}$ during the immobilization process. Furthermore, the structure of the non-close packed colloidal monolayers can be finely tuned by varying the ionic strength and geometric parameters between colloidal particles and nanomesh. Therefore one is able to specifically and selectively adsorb one or several particles into one individual nanohole.

\section{Introduction}

Colloidally assembled structures have been widely applied over the past years to prepare a broad range of functional nanoand mesostructured materials. ${ }^{1}$ Despite the fact that complex two- and three-dimensional ensembles can be fabricated by fairly simple methods, this research field is still very active due to the many degrees of freedom inherent in colloidal assemblies, such as packing geometry, inter-particle spacing, or particle material. ${ }^{2,3}$ For any preparation method under consideration, the tight control over the mutual colloidal interaction forces, the adsorption kinetics, and the drying conditions are of paramount importance in order to obtain the desired colloidal superstructure in two or three dimensions. These assemblies show many new properties in terms of their

\footnotetext{
${ }^{a}$ Physical Chemistry - Polymer Systems, University of Bayreuth, Universitätsstr. 30, 95447 Bayreuth, Germany. E-mail: Markus.Retsch@uni-bayreuth.de

${ }^{b}$ Physical Chemistry - Physics of Polymers, University of Bayreuth, Universitätsstr. 30, 95447 Bayreuth, Germany. E-mail: Georg.Papastavrou@uni-bayreuth.de $\dagger$ Electronic supplementary information (ESI) available: Photos and optical microscopy images, correlative optical and electron microscopy, low magnification SEM images, SEM images of adsorbed PMMA particles as well as $320 \mathrm{~nm}$ particles immobilized at $\mathrm{pH} 3$ and $\mathrm{pH} 12$, SEM images of particles assembled on hole arrays with variable center-to-center distances, radial distribution functions of $320 \mathrm{~nm}$ particles adsorbed at $\mathrm{pH} 3$ and variable ionic strengths, video of particles assembled under the confocal laser scanning microscope at different $\mathrm{pH}$ values, zeta potential measurements. See DOI: 10.1039/c6nr03113g
}

optical appearance, ${ }^{4,5}$ thermal conductance, ${ }^{6}$ or reflection properties, ${ }^{7,8}$ which can be tuned by varying the colloidal dimensions and order parameters. While these structures can extend in all three dimensions, it is often the first layer on the substrate that crucially defines the growing conditions of the following layers. ${ }^{9,10}$

In order to build 2-dimensional colloidal structures with maximal degrees of freedom, the underlying interactions governing the assembly of colloidal structures have to be finely controlled. Previous wet-chemical approaches can be classified as either convective or electrostatic assembly. ${ }^{2}$ In topographically controlled assembly structures a particular substrate pattern, which is commensurate or larger than the colloidal particle under consideration, is fabricated with a distinct height. Suitable patterns of various symmetry and periodicity have been produced for example by e-beam lithography or photolithography. The colloidal order is driven by capillary forces, which take place at the liquid-gas interfaces and drive the particle in the topographically lower regions of the pattern. Consequently, a dry colloidal monolayer or crystal is obtained at the end of the assembly process. The topographic template substrates provide access to hierarchical two-dimensional colloidal arrays, ${ }^{11-13}$ non-close packed ensembles, ${ }^{14,15}$ larger mono-crystalline domains, ${ }^{16,17}$ and predefined lattice symmetries. ${ }^{9,18,19}$ Additionally, very precise particle positioning on sparsely distributed topographic features has been demonstrated, which allows to prepare few oriented particle clusters on a flat substrate. ${ }^{20}$ 
Capillary forces commonly dominate electrostatic interaction between the particles during the drying procedure. ${ }^{21}$ However, in absence of such capillary forces, e.g. by transfer to a solvent with a lower surface tension ${ }^{22}$ the original structure remains intact. If the particle-substrate interaction is sufficient to prevent rearrangement of adsorbed particles, one finds the so-called extended random-sequential adsorption, ${ }^{23,24}$ which includes the inter-particle forces due to diffuse layer overlap. However, the single particle positions are stochastically distributed despite the presence of characteristic separation distance between the particles.

The electrostatic immobilization of colloidal particles on chemically modified patches, which are considerably larger than the particle itself is a commonly utilized strategy to obtain a deterministic placement of particles at defined places. $^{21,25-27}$ The necessary surface modification has been achieved by molecular self-assembled monolayers, ${ }^{25}$ polyelectrolytes, ${ }^{26}$ microcontact printing, ${ }^{21}$ or functional silanes. ${ }^{27}$ Nevertheless, the mutual order within these large area patches is still governed by random sequential adsorption or hexagonal packing in the case that capillary forces dominate.

In order to implement inter-particle spacing that show welldefined order parameters extending over nearest neighbours, different strategies have to be followed. One possibility is defined chemical modification in combination with highly specific interactions, such as receptor-ligand pairs or DNAhybridization. ${ }^{28,29}$ However, these preparation techniques require sophisticated equipment in order to prepare samples with sub-micron features.

Here, we present a more elegant method that is based on nanomesh structures, which are obtained by evaporation of noble metals on pre-adsorbed and etched colloidal monolayers. This so-called nanosphere lithography is well-established and has been described previously by various research groups. $^{30-34}$ Subsequently we orthogonally modify the two different surface materials (i.e. gold and glass) with thiols and silanes, respectively, in order to obtain highly defined surface areas of opposite charge. The term orthogonally expresses that this independent functionalization can provide surface areas with opposite characteristics, such as sign of surface charge. During particle deposition these surface charges on the structured collector surface lead to a defined adsorption of the particles that depends solely on the particle-surface interaction and not on inter-particle interaction. Hence, particle spacing superior to the ones obtainable by inter-particle forces can be achieved - yet at a high surface coverage. We demonstrate that one can obtain a highly selective process by which a defined number of small particles can be assembled in defined submicron surface areas. The required tuning of the particle/substrate and particle/particle interaction parameters can be achieved by adjustment of the ionic strength and the pH. We investigate the self-assembly process for pattern patches, which are larger, equal or smaller than the colloid size under investigation. The underlying interaction mechanisms during the particle deposition process have been quantified by direct force measurement based on the colloidal probe technique. ${ }^{35}$
In particular, direct force measurements demonstrate that the surface charge and not topographic features are responsible for the observed, irreversible adsorption process.

\section{Results and discussion}

In Fig. 1 the concept of selective particle deposition into the nanomesh is outlined. The different materials of the nanohole array allow an orthogonal functionalization of glass and gold. The glass holes are selectively functionalized with an aminosilane to obtain positively charged holes in acidic and neutral $\mathrm{pH}$ conditions, thus attracting the negatively charged polystyrene particles. By contrast, the Au-mesh is treated with an $\mathrm{OH}$-terminated thiol, which has been found to be negatively charged at $\mathrm{pH} 4 .^{36}$ This modification introduces a repulsive interaction while being hydrophilic and thus resulting in good wetting properties of the entire surface.

Although directed electrostatic adsorption of nanoparticles by chemically patterned surfaces has been shown before for $40 \mathrm{~nm} \mathrm{Au}$ colloids, ${ }^{29}$ a quantitative investigation of the underlying mechanism is still missing. For the first time, we directly determined the forces responsible for the particle adsorption by colloidal probe AFM measurements and studied the influence of particle size, $\mathrm{pH}$ and ionic strength in detail. Furthermore we extended the size range applicable to colloidal particles between $150 \mathrm{~nm}$ and $1500 \mathrm{~nm}$. Atomic force microscopy is utilized to study the long-range interactions on the orthogonally functionalized Au-nanohole arrays in order to understand particle immobilization on these substrates. By using a polystyrene (PS) bead as colloidal probe, static force measurements can be performed to directly reveal the interaction behaviour between the particles and the sample. This allows for a direct comparison of AFM measurements and macroscopic particle immobilization experiments. Exemplary force versus distance profiles on both, the Au-mesh and the $\mathrm{NR}_{4}{ }^{+}$-modified glass, are shown in Fig. 2 for measurements in $\mathrm{pH} 4$ und $\mathrm{pH} 10$ solution. When using $\mathrm{HCl}$ and $\mathrm{NaOH}$ to adjust the $\mathrm{pH}$ value, for $\mathrm{pH} 4$ and $\mathrm{pH} 10$ the ionic strength of the solution equals $0.1 \mathrm{mM}$. Thus, differences in the adsorption process can be directly referred to the $\mathrm{pH}$ value. All AFM measurements were conducted in aqueous solutions with a low total ionic strength of $0.2 \mathrm{mM}$ to achieve sufficiently large Debye lengths.

At pH 4 (Fig. $2 \mathrm{~b}$ and d) the force profiles on the approach part (red curve) significantly differ between the Au-mesh and

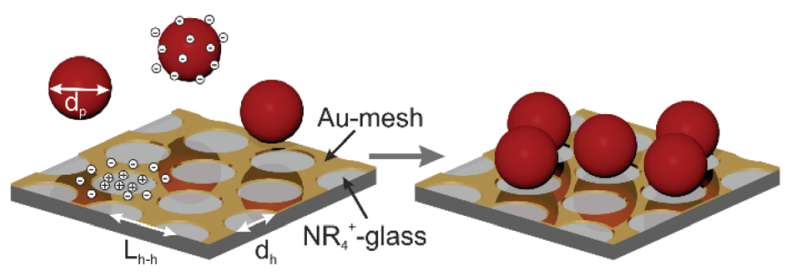

Fig. 1 Schematic representation of the self-assembly of polystyrene particles on a nanohole array. 
(a)

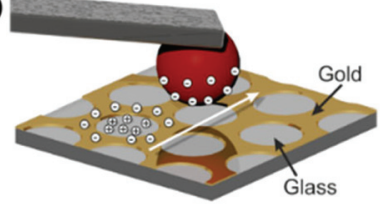

(b)

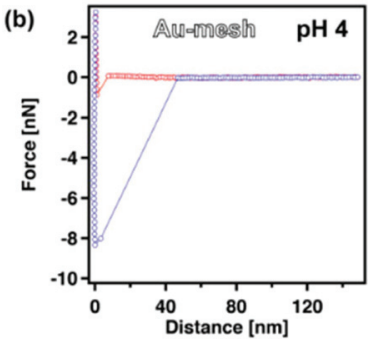

(d)

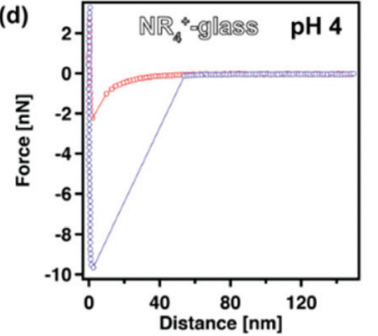

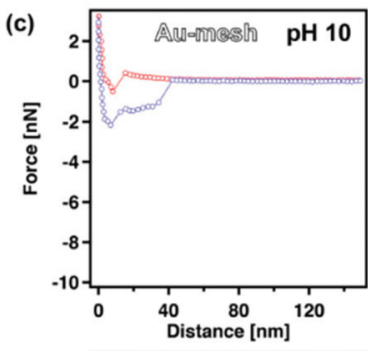

(e)

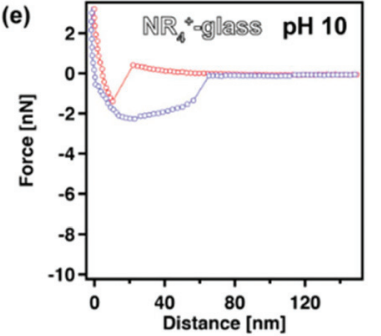

Fig. 2 (a) Schematic illustration of an AFM force experiment with a polystyrene colloidal probe on an Au-nanohole array. (b-e) Resulting force versus distance profiles for measurements in an aqueous solution at $\mathrm{pH} 4$ and $\mathrm{pH}$ 10. Depiction of the approach (red) and retraction (blue) part of force versus distance profiles on the Au-mesh (b, c) and $\mathrm{NR}_{4}{ }^{+}-$ glass (d, e).

the $\mathrm{NR}_{4}{ }^{+}$-glass, whereas the retraction parts (blue curve) show comparable trends for the adhesion once the particles are in contact with the surface. On the $\mathrm{NR}_{4}{ }^{+}$-modified glass a distinct long-range attraction for the polystyrene particles used as probe particles is observed. For the thiol-modified Au-surface, however, an absence of long-range attractive forces and a slight repulsion is observed. Particles adhere strongly at this $\mathrm{pH}$ to both surface types, suggesting an irreversible adsorption of particles once they are in contact with the surface.

At pH 10 (Fig. 2c and e) the long-range attraction forces between the cationic glass surface and the polystyrene probe vanish and instead a repulsive interaction is observed. Additionally, the repulsive interactions on the Au-surface areas increase. The combination of both effects leads to an effectively repulsive substrate for the negatively charged PS particles at $\mathrm{pH}$ 10. However, the retraction curves show again adhesion, albeit lower than for $\mathrm{pH}$ 4. Monolayers of hydroxy-terminated thiols are known to have a negative surface potential even in slightly acidic environment due to preferential accumulation of anions at the monolayer surface, while at $\mathrm{pH} 10$ an increased adsorption of hydroxyl ions and thus a more negative surface charge is reported. ${ }^{36-38}$ The weak repulsive forces at pH 10 for the aminosilane-modified glass can be attributed to hydroxyl-ion adsorption as well as an incomplete silane layer with an underlying highly negatively charged glass substrate.

Due to sulfate moieties, the particles are negatively charged over the whole $\mathrm{pH}$-range as confirmed by zeta potential measurements for three different particle sizes (Fig. S11†). Nevertheless, the magnitude of the zeta potential increases in alkaline conditions due to a higher dissociation of the sulfate moieties, leading to stronger substrate-particle repulsion.

The adhesion forces have found to be almost independent of the position on the nanohole array. Once a particle is in contact with the surface it adsorbs irreversibly. Based on these interaction profiles, we expect the long-range interaction forces to be the crucial key for a selective guidance of the particles into the holes.

A more detailed study of the local interaction forces is important for the rational understanding of the process of electrostatic focusing. Fig. 3a shows a topography image resulting from a force mapping experiment. The hexagonally patterned Au-mesh can be inferred from the topographic image with the black areas marking the glass surface. The thickness of the gold-mesh amounts to $\sim 45 \mathrm{~nm}$. The different symbols mark the position of individual force profiles resulting in a cross-section over one nanohole. The corresponding approach part of the force profiles is depicted in Fig. 3b. A clear trend of increasing attractive forces towards the center of the hole is observable. The attractive forces vanish at the edges and turn repulsive on the Au-mesh. Since the effective apex diameter of the probe is comparable to the hole dimensions, the gradual transition of the interaction forces may be the result of mixed contributions from both the Au-mesh and the $\mathrm{NR}_{4}{ }^{+}$-glass. Minor deviations from the trend may be attributed to a certain sample roughness. This line scan demonstrates that the particles will be exclusively attracted towards the glass surface, due to the electrostatic interaction, which we refer to as electrostatic focusing. We want to stress that these experiments have been carried out on completely water-submersed substrates. Therefore, contributions from capillary or convective forces can be ruled out. Also hydrophobic contributions will not play a role due to the hydrophilic moieties on the Au-mesh (hydroxyl-terminated) and glass surface (quarternary-amine terminated).

Based on the AFM force mapping experiments, we can now predict the macroscopic adsorption behavior of negatively (a)

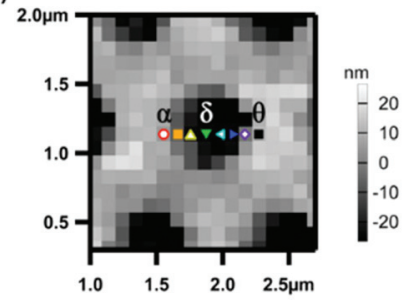

(b)

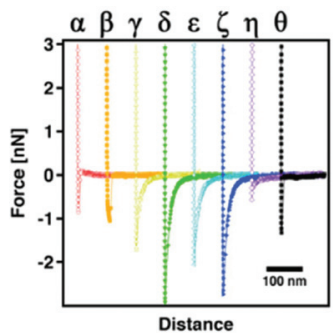

Fig. 3 (a) Topography image resulting from the force mapping experiment at $\mathrm{pH}$ 4. The symbols indicate the positions of a line scan of force profiles across one hole. (b) Approaching force versus distance profiles corresponding to the symbols in (a). 
charged PS beads. In the following we will demonstrate that the particle immobilization can be tuned by $\mathrm{pH}$ and ionic strength, as well as by the respective size ratios between particle, hole diameter, and lattice periodicity. Furthermore, we established a protocol allowing us to transfer this fully immersed system into the dry state.

\section{Tuning by pH}

In Fig. 4 the adsorption of PS spheres on functionalized $\mathrm{Au}$ nanomeshes is depicted in dependence of the $\mathrm{pH}$ value of the particle dispersion. The $\mathrm{pH}$ was adjusted to $\mathrm{pH} 5$ and $\mathrm{pH} 10$ with $\mathrm{HCl}$ and $\mathrm{NaOH}$, respectively. For all experiments nanomeshes with a center-to-center distance of $L_{\mathrm{h}-\mathrm{h}}=1.04 \mu \mathrm{m}$ and a hole diameter of $d_{\mathrm{h}}=0.87 \mu \mathrm{m}$ were used. The in-situ assembly dynamics of commercial fluorescent $606 \mathrm{~nm}$ PS particles into the nanomeshes can be monitored using confocal laser scanning microscopy. No further functionalization of the PS particles is necessary. A video showing the first $10 \mathrm{~min}$ of the assembly process for both $\mathrm{pH}$ values is shown in the ESI. $\dagger$ Fig. $4 \mathrm{a}$ and $\mathrm{b}$ display off-equilibrium snapshots of the adsorbed particles (green) $10 \mathrm{~min}$ after the addition of the particle dispersion on the nanomesh at pH 5 and pH 10, respectively. A clear correlation between the $\mathrm{pH}$ value of the particle dispersion and the particle adsorption can be recognized. At pH 5 a fast adsorption of the negatively charged particles predominantly on the $\mathrm{NR}_{4}{ }^{+}$-functionalized glass surface can be observed with $26 \%$ of the holes being occupied after $10 \mathrm{~min}$. Due to the electrostatic repulsion of the like-charged particles in solution, no particle agglomeration is observed and further
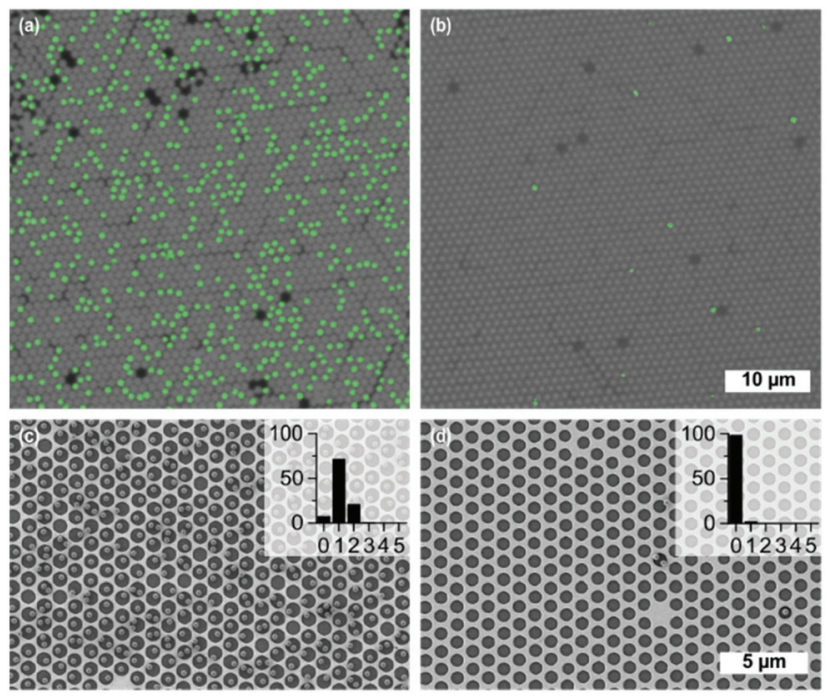

Fig. 4 Dependence of particle adsorption on the $\mathrm{pH}$. Top row: In situ confocal laser scanning microscopy of fluorescent polystyrene spheres (green) with a diameter of $606 \mathrm{~nm}$ at $\mathrm{pH} 5$ (a) and pH 10 (b) after $10 \mathrm{~min}$. Bottom row: SEM micrographs of $320 \mathrm{~nm}$ negatively charged PS particles on Au nanomeshes at pH 5 (c) and pH 10 (d). Insets in (c) and (d) show the frequency distribution of the number of particles per hole in percent. Both experiments were carried out with $1.04 \mu \mathrm{m} L_{\mathrm{h}-\mathrm{h}}$ and a hole diameter of $0.87 \mu \mathrm{m}$. particles are repelled once a hole is occupied. Moreover, the irreversible nature of the adsorption onto the glass areas is visible in the video, which supports the mechanistic results of the colloidal probe measurements. By contrast, at $\mathrm{pH}$ 10 hardly any particle adsorption can be found, with less than $1 \%$ occupied holes after $10 \mathrm{~min}$. The few particles on the surface show a rather undefined adsorption behavior and no clear tendency towards the glass surface.

This trend is consistent with the predictions from the force-distance measurements, which showed a repulsive force for both, the $\mathrm{NR}_{4}{ }^{+}$-functionalized glass and the hydroxy-functionalized $\mathrm{Au}$ surface at $\mathrm{pH}$ 10, resulting in no particle immobilization. At $\mathrm{pH} 4$, however, the extraordinary long-ranged electrostatic attraction at the glass surface drives the particles into the nanomesh holes. The same behavior can be seen in SEM images of nanomeshes after $15 \mathrm{~h}$ immersion in a dispersion of monodisperse $320 \mathrm{~nm}$ PS particles at pH 5 (Fig. 4c) and $\mathrm{pH} 10$ (Fig. 4d) and subsequent drying. After $15 \mathrm{~h}$ a plateau in the particle coverage is reached due to the particleparticle repulsion. Whereas the pH 5 sample is almost fully covered with selectively immobilized particles, practically no electrostatic adsorption takes place at $\mathrm{pH} 10$. The sample prepared at pH 5 exhibits a high occupation rate with more than $92 \%$ of the holes being occupied by one or more particles. Additionally, a high selectivity is discernible. Approximately $99 \%$ of the immobilized particles are trapped on the glass surface, which covers about $51.6 \%$ of the surface. Since high concentrations of colloidal dispersions ( $6 \mathrm{wt} \%$ ) were used for the colloidal assembly, this implies a self-quenching behavior of the particle adsorption process after the first particle layer. For $\mathrm{pH} 10$ the repulsive surface potential results in a very low surface coverage of less than 3.3 particles per $100 \mu^{2}$, which corresponds to only $2 \%$ occupied holes.

The transfer of the assembled particle structure into the dry state requires tight control on the capillary forces during the transfer process. Otherwise, the colloidal pattern formed in the wet state can be disrupted by strong capillary forces acting on liquid bridges between adjacent particles. Therefore, the solvent is systematically exchanged to first ethanol and then hexane prior to the drying step. ${ }^{22,39}$

The spatial uniformity of the dried samples can be seen in Fig. S1. $\uparrow$ Moreover, correlative optical microscopy and electron microscopy images of the $320 \mathrm{~nm}$ particles assembled at $\mathrm{pH} 5$ are shown in Fig. S2. $\dagger$ The samples are uniformly covered on a macroscopic scale on the range of several $\mathrm{mm}^{2}$ without drying effects and particle clusters. The good control of the capillary forces during the drying procedure can be inferred from the spatially separated beads, even within one mesh in the Au grid. Many of such particle pairs are separated by less than $100 \mathrm{~nm}$, yet, the strong adsorption to the glass surface prevented them from aggregating during drying. Thus, using an electrostatic adsorption mechanism instead of convective assembly, $^{20}$ a particle monolayer without direct interparticle contact even within the nanoholes is attained. The number of particles per hole for the corresponding sample is evaluated in the insets in Fig. $4 \mathrm{c}$ and d. To check the reproducibility of our 
method, two samples were prepared separately at equal conditions and compared in Fig. S3.† Both, the variation of the average number of particles per hole at different spots on one sample as well as the variation on different samples lies within the standard deviation of one particular spot.

To expand the influence of the $\mathrm{pH}$, additional samples were prepared at pH 3 (Fig. S4a†) and pH 12 (Fig. S4b†). pH 3 yields a nonselective adsorption of the particles onto the substrate. At this $\mathrm{pH}$ the repulsion of the Au surface is strongly reduced while the glass surface remains highly attractive. Therefore, the PS particles are strongly attracted towards the surface with approximately $36 \%$ becoming immobilized on the Au surface. Concomitant with the reduced $\mathrm{pH}$ is a reduction of the Debye screening length of the particles, which results in a higher loading of the particles on the surface and a decreased interparticle distance compared to $\mathrm{pH} 5$ (see Fig. S5†). Nevertheless, despite the high surface coverage no particle agglomeration but clearly separated spheres are noticeable. At pH 12 no significant difference can be seen compared to pH 10, with no adsorption being observable.

Overall, the choice of the $\mathrm{pH}$ of the particle dispersion is critical for the controlled immobilization. Weak acidic conditions are favorable to achieve high selectivity at a concomitantly high surface coverage.

\section{Tuning by ionic strength}

To separately investigate the contribution of the Debye length without the influence of the $\mathrm{pH}$, the ionic strength was varied by adding $\mathrm{NaCl}$ while $\mathrm{pH} 5$ was kept constant throughout the experiments. In Fig. 5 the adsorption of $320 \mathrm{~nm}$ PS particles into the $\mathrm{Au}$ nanomeshes is displayed for $\mathrm{NaCl}$ concentrations of $1 \mathrm{mM}$ (a), $10 \mathrm{mM}$ (b) and $100 \mathrm{mM}$ (c) and a constant assembly time of $15 \mathrm{~h}$. Low magnification SEM images are depicted in Fig. S6. $\dagger$

For an ionic strength of $1 \mathrm{mM}$, the average number of trapped particles is increased to 1.88 particles per hole compared to 1.15 particles per hole for the sample with an ionic strength of $<0.1 \mathrm{mM}$ (Fig. 4c). Nonetheless, a high selectivity is preserved with $99 \%$ of the particles being adsorbed on the glass surface. Increasing the ionic strength to $10 \mathrm{mM}$, even 3.22 particles per hole can be reached, while the selectivity suffers only by $3 \%$. Further increasing the ionic strength to $100 \mathrm{mM}$, however, results in a complete loss of selectivity (55\% of particles adsorbed on glass) and a random sequential
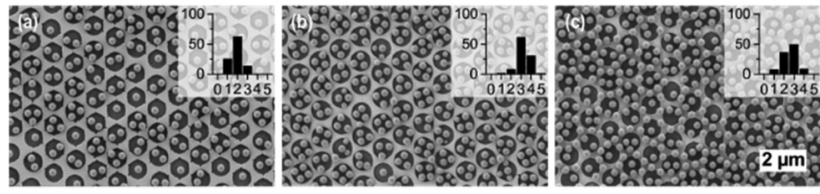

Fig. 5 Dependence of the adsorption of $320 \mathrm{~nm}$ negatively charged PS particles on the $\mathrm{NaCl}$ salt concentration on $\mathrm{Au}$ nanomeshes with $1.04 \mu \mathrm{m} L_{\mathrm{h}-\mathrm{h}}$ and a hole diameter of $0.87 \mu \mathrm{m}$. NaCl concentrations of $1 \mathrm{mM}$ (a), $10 \mathrm{mM}$ (b) and $100 \mathrm{mM}$ (c) were used. Insets show the frequency distribution of the number of particles per hole in percent.

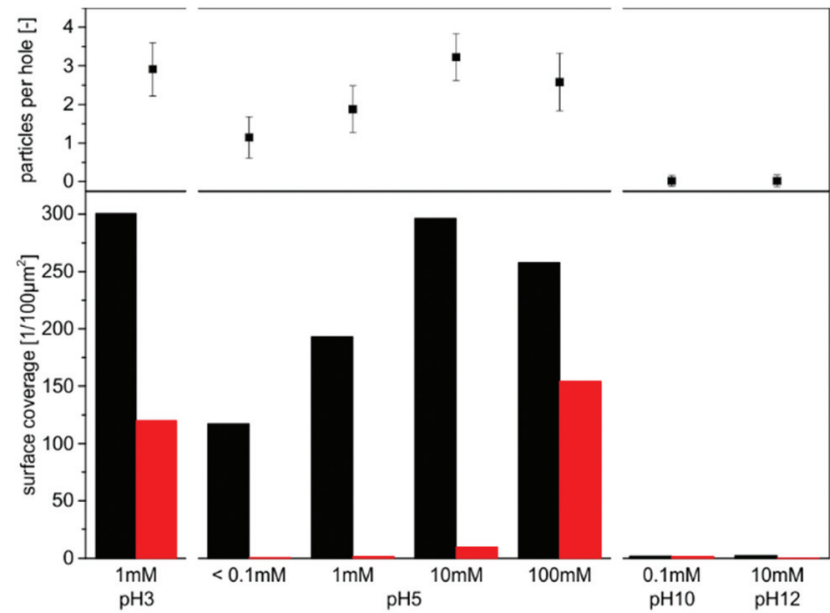

Fig. 6 Summary of the controlled deposition of $320 \mathrm{~nm}$ negatively charged PS particles on Au nanomeshes with $1.04 \mu \mathrm{m} L_{\mathrm{h}-\mathrm{h}}$ and a hole diameter of $0.87 \mu \mathrm{m}$. The area fraction of glass is $51.6 \%$. Average number of particles per hole (top) and surface coverage (bottom) of the samples in dependence of the different $\mathrm{pH}$ values and the overall ionic strengths. Red: particles on $\mathrm{Au}$, black: particles on glass.

adsorption of the PS particles is obtained. This trend can be corroborated with the nearest neighbor distance $\left(l_{\mathrm{NND}}\right)$ determined by the radial distribution functions, which correlates to the mutual distance between all trapped particles (Fig. S7 $\dagger$ ). Due to the increasing Debye screening, the $l_{\mathrm{NND}}$ slightly decreases for higher ionic strength from $447 \mathrm{~nm}$ for a salt concentration of less than $0.1 \mathrm{mM}$ to $382 \mathrm{~nm}$ for $100 \mathrm{mM}$. Yet, the particles are clearly separated and no agglomeration is visible even for high ionic strengths. One can also infer the selectivity of the adsorption process from the radial distribution function (RDF) profiles by the presence of clear correlation peaks at the positions corresponding to the hexagonally ordered nanomesh indicated by the vertical lines. The peaks are being smeared out with increasing ionic strength.

A summary regarding the selectivity and surface coverage of the particle adsorption for the case of $320 \mathrm{~nm}$ particles in $0.87 \mu \mathrm{m}$ holes at various $\mathrm{pH}$ values and salt concentrations is given in Fig. 6.

Increasing the ionic strength decreases the Debye screening length, and consequently leads to a higher amount of particles, which can be fitted into one single glass mesh. However, the selectivity is destroyed by too high salt concentrations $(100 \mathrm{mM})$. Meanwhile, changing the $\mathrm{pH}$ drastically reduces the mutual electrostatic interaction. The selectivity is already lost at an $\mathrm{HCl}$ concentration of $1 \mathrm{mM}(\mathrm{pH}$ ). Higher $\mathrm{pH}$ leads to strong repulsive forces on $\mathrm{Au}$ and glass resulting in almost no particle deposition.

\section{Tuning by geometry}

As the underlying nanomesh and the adsorbed particles are prepared independently, both the size of the particles and the geometry of the hole array can be tuned individually. Nevertheless, the size of the particles relative to the geometrical para- 
meters of the nanomesh has a significant influence on the adsorption process. In this case, we kept $L_{\mathrm{h}-\mathrm{h}}=1.04 \mu \mathrm{m}$ and $d_{\mathrm{h}}=0.87 \mu \mathrm{m}$ of the nanomesh constant and varied the particle diameter from $170 \mathrm{~nm}$ up to $1500 \mathrm{~nm}$ (see Fig. S9† for nanomeshes with different $L_{\mathrm{h}-\mathrm{h}}$ and $d_{\mathrm{h}}$ ). $\mathrm{pH} 5$ was used for the assembly as the particle dispersions in MilliQ water lead to $\mathrm{pH}$ 5 without the addition of $\mathrm{HCl}$ or $\mathrm{NaOH}$. Thus, the Debye lengths are not reduced by any electrolyte. All particles under investigation exhibited similar surface chemistry (see Table SI1 $\uparrow$ for zeta potential measurements). SEM images of the obtained structures are depicted in Fig. 7. Overview SEM images are depicted in Fig. S8. $\dagger$

A highly controlled adsorption is feasible over a wide range of particle diameters with high regioregularity up to a particle diameter of $1040 \mathrm{~nm}$. As the topographical contribution of the nanomesh can be neglected with respect to $d_{\mathrm{h}}$, a highly selective adsorption of nearly $100 \%$ is possible even for particle diameters that exceed $d_{\mathrm{h}}$ (Fig. 7e). Nonetheless, for particles with a diameter much larger than $d_{\mathrm{h}}$ (Fig. 7f) a loss of regularity is observed. This can be due to gravitational forces, which are more critical for larger particles. Moreover, as the particle dimensions are much greater than the surface structure, the particles may not recognize a strong chemical contrast between the $\mathrm{NR}_{4}{ }^{+}$-functionalized glass surface and the hydroxy-functionalized Au surface, reducing the possibility for electrostatic focusing.

The number of particles immobilized per hole is evaluated in the insets of Fig. 7. In Fig. 8a the average number of particles per hole is given as a function of the particle diameter. Apparently, single loading of the holes is only possible in a small size range between $0.5 d_{\mathrm{h}}$ and $d_{\mathrm{h}}$. Accordingly, for the particles with $570 \mathrm{~nm}$ (Fig. 7c) and $740 \mathrm{~nm}$ (Fig. 7d) excellent hole occupation with single spheres surpassing $99 \%$ is achieved. In contrast to previous works, ${ }^{27}$ we constricted the pattern size to the sub micrometer dimension allowing the production of non-close packed particle arrays with interparticle distances controlled by the template. Below the threshold of $0.5 d_{\mathrm{h}}$ multiple deposition of particles per hole is noticeable, with 1.15 particles per hole for a particle diameter of $320 \mathrm{~nm}$ and 2.11 particles per hole for a particle diameter of $166 \mathrm{~nm}$.

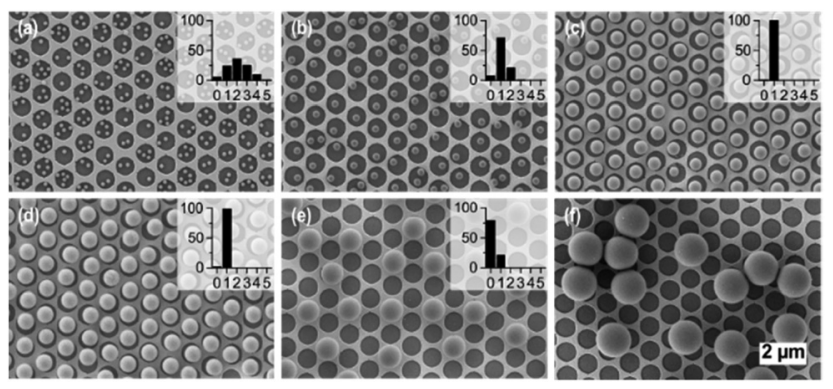

Fig. 7 PS particles of different diameter immobilized in nanohole arrays with $d_{\mathrm{h}}=0.87 \mu \mathrm{m}$ and $L_{\mathrm{h}-\mathrm{h}}=1.04 \mu \mathrm{m}$. PS particles have a diameter of (a) $166 \mathrm{~nm}$ (b) $320 \mathrm{~nm}$ (c) $570 \mathrm{~nm}$ (d) $740 \mathrm{~nm}$ (e) $1040 \mathrm{~nm}$, and (f) $1500 \mathrm{~nm}$. Insets show the frequency distribution of the number of particles per hole in percent.
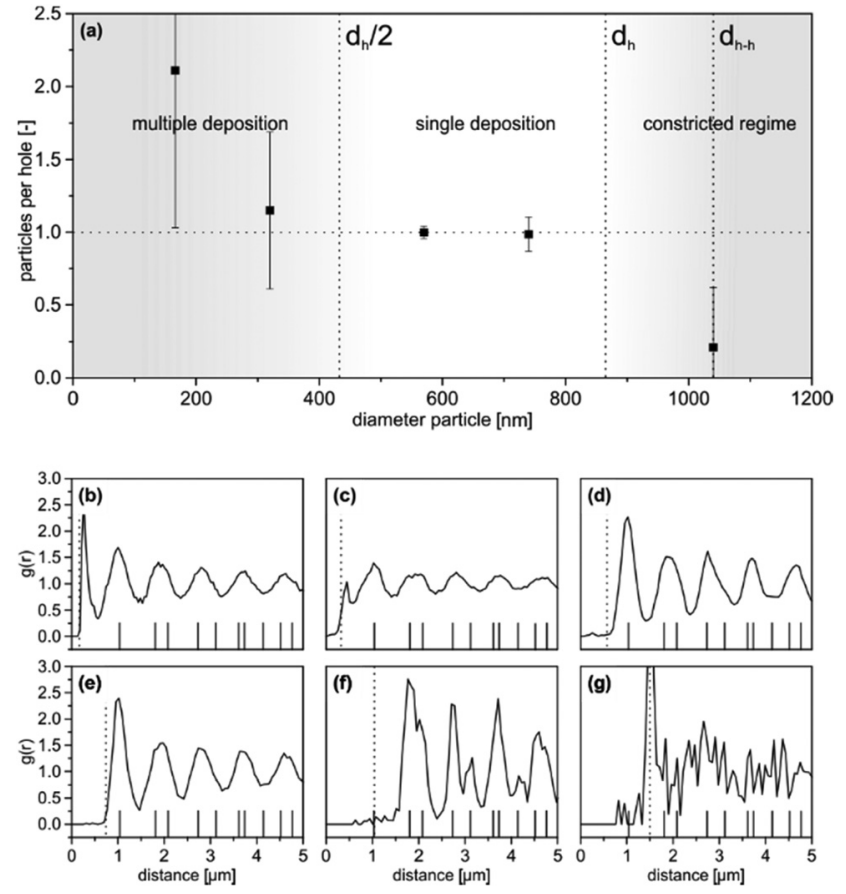

Fig. 8 (a) Particle coverage as a function of particle diameter. Vertical dashed lines mark important geometric transition regions. Radial distribution functions $g(r)$ for various particles immobilized in a nanomesh with $1.04 \mu \mathrm{m} L_{\mathrm{h}-\mathrm{h}}$ and hole diameter $d_{\mathrm{h}}=0.87 \mu \mathrm{m}$ : (b) $166 \mathrm{~nm}$ (c) $320 \mathrm{~nm}$ (d) $570 \mathrm{~nm}$ (e) $740 \mathrm{~nm}$ (f) $1040 \mathrm{~nm}$ (g) $1500 \mathrm{~nm}$. The dotted lines indicate the particle diameter, the solid ticks indicate the $g(r)$ of the underlying Au-nanomesh.

Furthermore, particles below this threshold will always exhibit a more or less broad distribution of particles trapped per hole, depending on the ionic strength of the particle dispersion as discussed above.

When the particle diameter exceeds $d_{\mathrm{h}}$ and is in the range of the lattice parameter of the nanomesh, the adsorption process is constricted by the interparticle electrostatic repulsion. Particles with a diameter of $1040 \mathrm{~nm}$ (Fig. 6f), which is equal to $L_{\mathrm{h}-\mathrm{h}}$, do not occupy adjacent holes as this would imply unfavorable direct contact of the particles. This results in a theoretical maximum of 0.25 particles per hole assuming that particles cannot be trapped in adjacent spots once a particle is immobilized in one hole. In practice, a number of approximately 0.21 particles per hole is reached as the random fashion of the adsorption process is taken into account.

To accomplish an occupation rate approaching $100 \%$ with these large spheres, however, $L_{\mathrm{h}-\mathrm{h}}$ has to be increased (Fig. S9c $\dagger$ ).

For a quantitative analysis of the obtained, two-dimensional colloidal patterns, the center-of-mass coordinates of the adsorbed particles were determined in ImageJ and used to calculate the radial distribution function $g(r)$ (Fig. 8b). This function displays the frequency of the occurring particle-particle distances. The presence of defined peaks up to high distances corresponding to the most likely distances occurring in the pattern indicates a system with good long-range order. 
Assumptions about the short-range order can be made by evaluating the position and width of the first peak $\left(l_{\mathrm{NND}}\right)$. The solid ticks indicate the peak positions of the given ideal hexagonal structure of the underlying nanomesh with the first tick representing $L_{\mathrm{h}-\mathrm{h}}$. For all particles up to a diameter of $1040 \mathrm{~nm}$ short-range order is detectable, with a well-defined $l_{\mathrm{NND}}$ and a low value of $g(r)$ at $d_{\mathrm{p}}$ (dashed line) indicating the absence of particle agglomeration. Moreover, long-range order is visible for all particles except the $1500 \mathrm{~nm}$ particles. The predominant occupation of the glass surface results in clear peaks occurring at the distances corresponding to the hexagonal lattice of the hole array.

The particles with a diameter of $166 \mathrm{~nm}$ (Fig. 8b) and $320 \mathrm{~nm}$ (Fig. 8c) exhibit an $l_{\mathrm{NND}}$ of $270 \mathrm{~nm}$ and $447 \mathrm{~nm}$, respectively, which is smaller than $L_{\mathrm{h}-\mathrm{h}}$ but greater than $d_{\mathrm{p}}$ as multiple particles are trapped per hole. For the particle diameters of $570 \mathrm{~nm}$ (Fig. 8d) and $740 \mathrm{~nm}$ (Fig. 8e) $g(r)$ is congruent with the $g(r)$ of the hole array with the $l_{\mathrm{NND}}$ equal to $L_{\mathrm{h}-\mathrm{h}}$. The relatively high standard deviation of the peaks despite of the single loading of the holes is given by the random, noncentrosymmetric position of the immobilized spheres.

No occupation of $L_{\mathrm{h}-\mathrm{h}}$ is noticeable for a particle diameter of $1040 \mathrm{~nm}$ (Fig. 8f). Instead, $l_{\mathrm{NND}}$ of the particles is present at the preferred second distance of the nanomesh lattice. However, a particular well-defined long-range order is observed at higher distances due to the uniformly centered position of the particles.

A different case is discernible for the particle diameter of $1500 \mathrm{~nm}$ (Fig. 8g). The maximum of $g(r)$ at $d_{\mathrm{p}}$ correlates to the presence of direct interparticle contacts. Furthermore, no longrange order is inferable, which associates with random sequential adsorption.

Finally, the adsorption process is highly reproducible not only for a broad range of particle diameters but universally applicable for negatively charged polymer colloids. This generality of the method is depicted in Fig. S10 $\uparrow$ for colloidal PMMA particles with a diameter of $260 \mathrm{~nm}$. As the self-assembly process simply relies on electrostatic interactions, the concept can be readily conveyed to arbitrary negatively stabilized colloids. Therefore, a sophisticated surface functionalization of the particles is not necessary.

\section{Conclusion}

In summary, we investigated the controlled placement of polystyrene particles into $\mathrm{Au}$ nanohole arrays by electrostatic modelling of the substrate. An orthogonal functionalization of gold and glass is exploited to introduce a selective electrostatic attraction of the particles towards the glass surface. In this study, we demonstrate the highly reproducible immobilization of polystyrene particles over a broad range of particle diameters ranging from $170 \mathrm{~nm}$ to $1040 \mathrm{~nm}$. Tailoring the ratio of the diameter of the particles and the geometric parameter of the nanohole arrays enables one to switch between different adsorption regimes, with multiple particles per hole or highly defined single particle adsorption.

Furthermore, we found that the tuning of the experimental parameters such as $\mathrm{pH}$ and ionic strength during the assembly process is important to control the adsorption pattern. Colloidal probe atomic force microscopy revealed a high sensitivity of the effective interaction potentials towards the $\mathrm{pH}$. The attractive potential of the $\mathrm{NR}_{4}{ }^{+}$-functionalized glass can be switched off when increasing the $\mathrm{pH}$ from $\mathrm{pH} 3$ to $\mathrm{pH} 10$ giving a uniform repulsive potential and an impeded particle adsorption. In addition, the ionic strength of the particle dispersion determines the Debye lengths of the electrostatic forces deriving from the nanomesh and the particles. Thus, the average number of particles in one hole can be controlled by ionic strength maintaining a good selectivity up to $10 \mathrm{mM}$ $\mathrm{NaCl}$. Higher salt concentrations result in random adsorption of the particles. By adjusting an ionic strength of $10 \mathrm{mM}$ the number of particles per hole was nearly tripled.

This selective particle deposition occurs foremost in the dispersed liquid state without the aid of topography, convective or capillary forces. Confocal microscopy hints towards an irreversible adsorption of the particles onto the glass surface, which is supported by adhesive profiles in the retracting curves of colloidal probe force measurements. We could successfully transfer this immersed structure into the dry state by exchanging the dispersion medium and thereby controlling the capillary forces. Finally, this structuring concept works universally for polymer particles with a negative surface charge.

To our knowledge, this is the first paper, in which the immobilization of particles is shown in nanohole arrays made by bottom-up nanosphere lithography. The electrostatic adsorption allows to direct particles into hole arrays with very shallow topographic contrast, which are not accessible with convective or capillary particle assembly. This represents a simple and versatile route for the assembly of particles on optically active metal substrates, which might be utilized to create complex plasmonic waveguide structures or sensing devices in the future.

\section{Experimental section}

\section{Materials}

Polystyrene particles were purchased at Microparticles $\mathrm{GmbH}$ (Berlin) or synthesized using emulsifier free emulsion polymerization. $\quad N$-Trimethoxysilylpropyl- $N, N, N$-trimethylammonium chloride (ABCR GmbH, Karlsruhe, Germany, 50\% in methanol), 3-mercapto-1-propanol (ABCR GmbH, Karlsruhe, Germany, >96\%), sodium dodecyl sulfate (Serva Electrophoresis GmbH, Heidelberg, Germany), Hellmanex III (Hellma GmbH, Müllheim, Germany), ethanol (Sigma-Aldrich GmbH, Munich, Germany, $\geq 99.8 \%$ ), $n$-hexane (VWR International GmbH, Darmstadt, Germany, >98\%), NaCl (Sigma-Aldrich GmbH, Munich, Germany, $\geq 99.0 \%$ ), $\mathrm{NaOH}$ (Sigma-Aldrich GmbH, Munich, Germany, $\geq 98 \%$ ), ammonium hydroxide solu- 
tion (Sigma-Aldrich GmbH, Munich, Germany, 28.0-30.0\%), $\mathrm{HCl}$ (Grüssing GmbH, Filsum, Germany, $1 \mathrm{M}$ ) were used as received. Standard microscopy slides (Menzel, Braunschweig, Germany) were used.

\section{Fabrication of Au nanomeshes}

Monolayers were prepared according to the procedure of Retsch et $a .^{40}$ In brief cationically functionalized glass slides were spin cast with a $3 \mathrm{wt} \%$ particle dispersion $(1.04 \pm 0.04 \mu \mathrm{m}$ diameter) at a speed of $4000 \mathrm{rpm}$. Freely floating monolayers were assembled at an air/water interface by slow immersion of the particle coated glass substrate into a $0.1 \mathrm{mM}$ SDS solution in MilliQ water. The aqueous phase was adjusted to $\mathrm{pH} 12$ by adding a few drops of $\mathrm{NH}_{3}$. The floating monolayer was finally transferred to a glass substrate and dried in air.

The prepared monolayers were etched in a plasma reactor MiniFlecto (Plasma Technology GmbH, Herrenberg, Germany) with $75 \%$ argon and $25 \%$ oxygen at $80 \mathrm{~W}$ power at a pressure of 0.14 mbar. Etching was conducted for $20 \mathrm{~min}$ in order to obtain non-close packed monolayers with particles of $870 \mathrm{~nm}$ diameter.

A $3 \mathrm{~nm}$ chromium layer and $50 \mathrm{~nm} \mathrm{Au}$ were deposited using a Balzers BA360 thermal evaporation chamber. The layer thickness was monitored via a SQM 160 microbalance (Sigma Instruments, Schaefer Technologie $\mathrm{GmbH}$ ). The particles were removed using Scotch ${ }^{\circledR}$ tape $(3 \mathrm{M})$ giving the nanohole arrays. The $\mathrm{Au}$ substrates were cleaned for $10 \mathrm{~min}$ in an ultrasonic bath with a $2 \%$ aqueous Hellmanex (Hellma GmbH, Mühlheim, Germany) solution in MilliQ water. The surfactant was extensively rinsed off with MilliQ water and the substrates were placed in the ultrasonic bath in ethanol for 10 minutes and dried with compressed air.

\section{Chemical functionalization of Au nanomeshes}

After hydrophilization in $100 \%$ oxygen plasma at 0.2 mbar for $30 \mathrm{~s}$ the nanohole arrays were immersed in a 1 vol\% solution of $N$-trimethoxysilylpropyl- $N, N, N$-trimethylammonium chloride in MilliQ water for 1 hour. Excess silane was rinsed off with MilliQ water and the substrates were placed in a 1 vol\% solution of 3-mercapto-1-propanol in ethanol for 2 hours. After rinsing with ethanol the substrates were dried at $90^{\circ} \mathrm{C}$ for 1 hour.

\section{Particle immobilization on nanomeshes}

The nanohole arrays were placed in the particle dispersion for at least 2 hours. The $\mathrm{pH}$ was adjusted with $\mathrm{HCl}$ and $\mathrm{NaOH}$, respectively. To remove excess particles, the supernatant was repeatedly diluted with MilliQ water. Care was taken that the substrates do not run dry during this rinsing procedure. Finally, the solvent was exchanged consecutively with ethanol and hexane and the sample was dried in air.

\section{Characterization}

Confocal Laser Scanning Microscopy was performed on a TSC SP8 STED $3 \times$ (Leica) using an oil immersion objective $(63 \times$ augmentation), a $514 \mathrm{~nm}$ laser for excitation and 4 frames per second. SEM images were taken on a LEO 1530 Gemini Field
Emmission SEM (Carl Zeiss AG, Oberkochen, Germany) at 3.00 $\mathrm{kV}$. The images were evaluated with the software ImageJ. ${ }^{41}$ Particle positions were extracted using the "Find Maxima" tool and the radial distribution functions were calculated using a self-written software (details are given in the ESI $\dagger$ ). For the statistical analysis the occupation of at least 450 holes was evaluated for each sample, i.e. 1000-2000 particles were analyzed for the $151 \mathrm{~nm}-1040 \mathrm{~nm}$ particles. 406 particles were analyzed for the particles with a diameter of $1500 \mathrm{~nm}$ (random adsorption). For the particles assembled at pH 10 and pH 12, 85 and 53 particles were counted, respectively.

The preparation of colloidal probes used here, has been described previously. ${ }^{38,42}$ Tipless AFM cantilevers (NSC-12, MikroMasch, Tallinn, Estonia) were cleaned with a series of solvents (ethanol, water, aceton) directly before preparation. A single polystyrene particle with an average diameter of $3.00 \mu \mathrm{m}$ (micro particles, Berlin, Germany) was attached to the end of a tipless cantilever by means of a micromanipulator (STM3, Märzhäuser, Wetzlar, Germany), which was mounted next to a fixed stage optical microscope (Axio Examiner D1, Zeiss, Jena, Germany). The particle was immobilized by UV-curable glue (Norland Optical Adhesive 63, Norland Products, Cranbury, NJ) that was cured directly on the microscope stage by means of the mercury lamp illumination. The spring constants of the cantilevers has been determined previously by the thermal noise method, ${ }^{43}$ i.e. before gluing the colloidal particles onto the lever.

Measurements of interaction forces were performed with an AFM equipped with a closed-loop scanner for all three axes (MFP-3D, Asylum Research, Santa Barbara, CA). The AFM was placed in an acoustic shielding. Force curves were acquired with a cantilever velocity of about $200 \mathrm{~nm} \mathrm{~s}^{-1}$ and a maximum loading force of about $5 \mathrm{nN}$. The surface topography was calculated from the set of consecutive force curves acquired on a grid with $3 \times 3 \mu \mathrm{m}$ size consisting of $30 \times 30$ points, which results in a surface map where the single data points have a separation of $100 \mathrm{~nm}$. The measured deflection versus displacement curves were converted to force versus distance curves by means of a set of custom evaluation procedure written in IGOR PRO (Wavemetrics, Portland, OR). ${ }^{42,44}$ The direct force measurements were performed in aqueous solution at $\mathrm{pH} 4$ and 10 and a total ionic strength of $0.2 \mathrm{mM}$.

\section{Acknowledgements}

This work was funded by the German Research Foundation (DFG) by the SFB840. C. S. acknowledges support from the Elite Network Bavaria (ENB). The experimental support by Tanja Feller is appreciated. M. R. acknowledges support by the VCI. The authors thank Fabian Nutz for the particle synthesis, Pia Ruckdeschel for correlative SEM measurements, Dr Martin Dulle for help with the $g(r)$ calculations and Dr Beate Förster and Martina Heider from the Bayreuth Institute of Macromolecular Research (BIMF) for their support using the scanning electron microscopy facilities. Prof. Stephan Förster is acknowledged for providing access to the confocal laser scan- 
ning microscope. Microparticles $\mathrm{GmbH}$ is acknowledged for providing special PS microbeads for the colloidal probe preparation.

\section{Notes and references}

1 O. D. Velev and S. Gupta, Adv. Mater., 2009, 21, 1897-1905.

2 N. Vogel, M. Retsch, C. A. Fustin, A. Del Campo and U. Jonas, Chem. Rev., 2015, 115, 6265-6311.

3 J. Zhang, Y. Li, X. Zhang and B. Yang, Adv. Mater., 2010, 22, 4249-4269.

4 M. Harun-Ur-Rashid, A. Bin Imran, T. Seki, M. Ishii, H. Nakamura and Y. Takeoka, ChemPhysChem, 2010, 11, 579-583.

5 N. Vogel, S. Utech, G. T. England, T. Shirman, K. R. Phillips, N. Koay, I. B. Burgess, M. Kolle, D. A. Weitz and J. Aizenberg, Proc. Natl. Acad. Sci. U. S. A., 2015, 112, 10845-10850.

6 F. A. Nutz, P. Ruckdeschel and M. Retsch, J. Colloid Interface Sci., 2015, 457, 96-101.

7 C. Stelling, C. Bernhardt and M. Retsch, Macromol. Chem. Phys., 2015, 216, 1682-1688.

8 N. Vogel, S. Goerres, K. Landfester and C. K. Weiss, Macromol. Chem. Phys., 2011, 212, 1719-1734.

9 A. vanBlaaderen, R. Ruel and P. Wiltzius, Nature, 1997, 385, 321-324.

10 J. Zhang, A. Alsayed, K. H. Lin, S. Sanyal, F. Zhang, W.-J. Pao, S. K. Balagurusamy, P. A. Heiney and A. G. Yodh, Appl. Phys. Lett., 2002, 81, 3176-3178.

11 Y. D. Yin, Y. Lu, B. Gates and Y. N. Xia, J. Am. Chem. Soc., 2001, 123, 8718-8729.

12 M. Müller, M. Karg, A. Fortini, T. Hellweg and A. Fery, Nanoscale, 2012, 4, 2491-2499.

13 X. Li, Y. Wu, L. Hang, D. Men, W. Cai and Y. Li, J. Mater. Chem. C, 2015, 3, 51-57.

14 M. Allard, E. H. Sargent, P. C. Lewis and E. Kumacheva, Adv. Mater., 2004, 16, 1360-1364.

15 V. Sharma, D. Xia, C. C. Wong, W. C. Carter and Y.-M. Chiang, J. Mater. Res., 2011, 26, 247-253.

16 L. Mishchenko, B. Hatton, M. Kolle and J. Aizenberg, Small, 2012, 8, 1904-1911.

17 I. Bita, J. K. Yang, Y. S. Jung, C. A. Ross, E. L. Thomas and K. K. Berggren, Science, 2008, 321, 939-943.

18 G. A. Ozin and S. M. Yang, Adv. Funct. Mater., 2001, 11, 95-104.

19 J. Hoogenboom, A. van Langen-Suurling, J. Romijn and A. van Blaaderen, Phys. Rev. Lett., 2003, 90.

20 L. Malaquin, T. Kraus, H. Schmid, E. Delamarche and H. Wolf, Langmuir, 2007, 23, 11513-11521.
21 J. Aizenberg, P. V. Braun and P. Wiltzius, Phys. Rev. Lett., 2000, 84, 2997-3000.

22 L. Isa, K. Kumar, M. Muller, J. Grolig, M. Textor and E. Reimhult, ACS Nano, 2010, 4, 5665-5670.

23 Z. Adamczyk and P. Warszyński, Adv. Colloid Interface Sci., 1996, 63, 41-149.

24 M. Semmler, E. K. Mann, J. Rička and M. Borkovec, Langmuir, 1998, 14, 5127-5132.

25 J. Tien, A. Terfort and G. M. Whitesides, Langmuir, 1997, 13, 5349-5355.

26 K. M. Chen, X. Jiang, L. C. Kimerling and P. T. Hammond, Langmuir, 2000, 16, 7825-7834.

27 U. Jonas, A. del Campo, C. Krüger, G. Glasser and D. Boos, Proc. Natl. Acad. Sci. U. S. A., 2002, 99, 5034-5039.

28 C. H. Lalander, Y. Zheng, S. Dhuey, S. Cabrini and U. Bach, ACS Nano, 2010, 4, 6153-6161.

29 Y. Zheng, C. H. Lalander, T. Thai, S. Dhuey, S. Cabrini and U. Bach, Angew. Chem., Int. Ed., 2011, 50, 4398-4402.

30 S.-M. Yang, S. G. Jang, D.-G. Choi, S. Kim and H. K. Yu, Small, 2006, 2, 458-475.

31 A. J. Morfa, E. M. Akinoglu, J. Subbiah, M. Giersig and P. Mulvaney, J. Appl. Phys., 2013, 114, 054502.

32 Y. Yu and G. Zhang, in Updates in Advanced Lithography, ed. S. Hosaka, InTech, 2013, ch. 1, pp. 3-34, DOI: 10.5772/56576, http://www.intechopen.com/books/updatesin-advanced-lithography/colloidal-lithography.

33 F. Burmeister, C. Schäfle, T. Matthes, M. Böhmisch, J. Boneberg and P. Leiderer, Langmuir, 1997, 13, 29832987.

34 Y. N. Xia, J. A. Rogers, K. E. Paul and G. M. Whitesides, Chem. Rev., 1999, 99, 1823-1848.

35 M. Kappl and H.-J. Butt, Part. Part. Syst. Charact., 2002, 19, 129.

36 P. Maroni, F. J. Ruiz-Cabello and A. Tiraferri, Soft Matter, 2014, 10, 9220-9225.

37 H. J. Kreuzer, R. L. Wang and M. Grunze, J. Am. Chem. Soc., 2003, 125, 8384-8389.

38 V. Kuznetsov and G. Papastavrou, J. Phys. Chem. C, 2014, 118, 2673-2685.

39 M. A. Ray and L. Jia, Adv. Mater., 2007, 19, 2020-2022.

40 M. Retsch, Z. Zhou, S. Rivera, M. Kappl, X. S. Zhao, U. Jonas and Q. Li, Macromol. Chem. Phys., 2009, 210, 230241.

41 W. S. Rasband, ImageJ, http://imagej.nih.gov/ij/.

42 S. Rentsch, R. Pericet-Camara, G. Papastavrou and M. Borkovec, Phys. Chem. Chem. Phys., 2006, 8, 2531-2538.

43 J. L. Hutter and J. Bechhoefer, Rev. Sci. Instrum., 1993, 64, 1868.

44 R. Pericet-Camara, G. Papastavrou, S. H. Behrens and M. Borkovec, J. Phys. Chem. B, 2004, 108, 19467-19475. 